\title{
Validação do Instrumento de Percepção do Acesso a Informação Compartilhada entre Equipe-Família em unidade neonatal
}

\author{
Validation of the Instrument for Perception of Access to Shared Information between Healthcare Team-Family in \\ neonatal unit \\ Validación del Instrumento de Percepción de Acceso a Información Compartida entre Equipo del Salud-Familia en \\ unidad neonatal

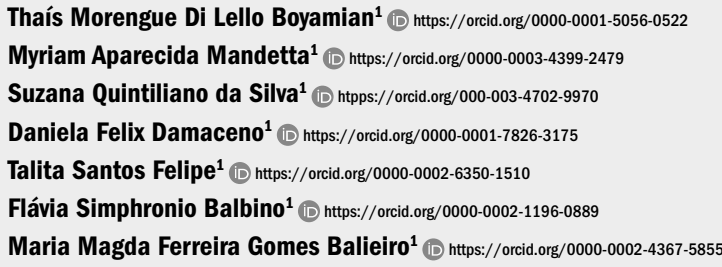

\section{Resumo}

Objetivo: Realizar a validação do Instrumento de Medida da Percepção da Família sobre o Acesso à Informação na Unidade Neonatal.

Métodos: Estudo metodológico, realizado com 85 representantes de famílias de recém-nascidos internados há mais de 72 horas na unidade neonatal. Os dados foram analisados pela psicometria e análise inferencial.

Resultados: A validação do conteúdo do instrumento por especialistas obteve concordância acima de $80 \%$, Alfa de Cronbach de 0,86 e na análise fatorial gerou oito fatores, cuja variabilidade total foi de 78,2\%. 0 escore médio da percepção do acesso à informação pela família foi 3,37 - quase sempre.

Conclusão: 0 instrumento obteve uma ótima consistência interna, cargas fatoriais que permitiram a manutenção dos itens nos instrumentos; e a família percebe que quase sempre têm acesso à informação, o que requer investimento na mudança da cultura organizacional para a valorização e inclusão da família.

\footnotetext{
Abstract

Objective: To carry out the validation of the Instrument for Measuring the Perception of the Family on Access to Information in the Neonatal Unit.

Methods: Methodological study, carried out with 85 representatives of newborn families hospitalized for more than 72 hours in the neonatal unit. Data were analyzed by psychometrics and inferential analysis.

Results: The validation of the instrument content by experts obtained agreement above $80 \%$, Cronbach's alpha of 0.86 and in the factor analysis it generated eight factors, whose total variability was $78.2 \%$. The average score for the perception of access to information by the family was 3.37 - almost always.

Conclusion: The instrument obtained great internal consistency, factor loadings that allowed the maintenance of items in the instruments; and the family realizes that they almost always have access to information, which requires investment in changing the organizational culture to value and include the family.
}

\section{Resumen}

Objetivo: Realizar la validación del Instrumento de Medición de la Percepción de la Familia sobre el Acceso a la Información en la Unidad Neonatal.

Métodos: Estudio metodológico, realizado con 85 representantes de familias de recién nacidos hospitalizados por más de 72 horas en la unidad neonatal. Los datos fueron analizados por psicometría y análisis inferencial. Resultados: La validación del contenido del instrumento por expertos obtuvo concordancia superior al $80 \%$, alfa de Cronbach de 0,86 y en el análisis factorial generó ocho factores, cuya variabilidad total fue del $78,2 \%$. El puntaje promedio para la percepción de acceso a la información por parte de la familia fue de 3.37 - casi siempre. Conclusión: El instrumento obtuvo gran consistencia interna, cargas factoriales que permitieron el mantenimiento de ítems en los instrumentos; y la familia se da cuenta de que casi siempre tienen acceso a la información, lo que requiere invertir en cambiar la cultura organizacional para valorar e incluir a la familia.

\section{Como citar:}

Boyamian TM, Mandetta MA, Silva SQ, Damaceno DF, Felipe TS, Balbino FS, et al. Validação do Instrumento de Percepção do Acesso a Informação Compartilhada entre Equipe-Família em unidade neonatal. Rev Soc Bras Enferm Ped. 2021;21(2):150-8.

\section{Descritores}

Enfermagem neonatal; Acesso a informação; Família; Unidade de terapia intensiva neonatal; Enfermagem pediátrica

\section{Keywords}

Neonatal nursing; Access to information; Family; Intensive care units neonatal; Pediatric nursing

\section{Descriptores}

Enfermería neonatal; Acceso a la información; Familia; Unidades de cuidado intensivo neonatal; Enfermería pediátrica

\footnotetext{
${ }^{1}$ Escola Paulista de Enfermagem, Universidade Federal de São Paulo, São Paulo, SP, Brasil.

Conflitos de interesse: nada a declarar.

Submetido: 1 de Outubro de 2021 | Aceito: 13 de Dezembro de 2021

Autor correspondente: Maria Magda Ferreira Gomes Balieiro | E-mail: mmfgbalieiro@unifesp.br

DOI: http://dx.doi.org/10.31508/1676-379320210021
} 


\section{Introdução}

Os períodos perinatal e neonatal são importantes momentos para os pais na construção da parentalidade, sendo, portanto, um grande evento na vida de uma família. $\mathrm{O}$ vínculo afetivo com a criança pode se iniciar mesmo antes da gestação e vai se solidificando com a gestação, o nascimento e durante o período pós-natal. A família como um todo tem de se reestruturar para assumir novos papéis, ajustar mudanças de comportamentos e responsabilidades pela vinda de um novo membro. ${ }^{(1)}$

No entanto, quando ocorre o nascimento de uma criança que requer hospitalização, a família vivencia sofrimento e estresse. ${ }^{(2)}$ Esta situação pode dificultar o contato espontâneo entre pais e recém-nascidos, requerendo o estabelecimento de uma boa comunicação entre família e a equipe, além de suporte para a construção da parentalidade. . $^{(3,4)}$

Percebe-se que apesar das evidências do acesso livre da família no ambiente da unidade neonatal para a saúde do $R N,{ }^{(5,6)}$ a sua participação ainda é limitada em decorrência de crenças restritivas e valores dos profissionais de saúde, da cultura institucional e da estrutura física dessas unidades, que ainda não aceitam a permanência e nem a participação dos pais/responsáveis nas vinte e quatro horas. ${ }^{(7)}$

O Modelo do Cuidado Centrado na Família (CCF) compreende a família como central na vida e no cuidado de seus membros $\mathrm{O}$ isolamento social da família é um fator de risco, sobretudo para os mais dependentes de cuidados, como as crianças, idosos e aqueles com doença crônica. ${ }^{(8)}$ Deste modo recomenda-se que os profissionais promovam a continuidade da ligação natural entre a família, seus pacientes e rede de apoio. Os pressupostos centrais do CCF são dignidade e respeito, em que as escolhas do paciente e família são ouvidas e respeitadas pelos profissionais de saúde, incorporando também seus conhecimentos, valores, crenças e cultura no planejamento e prestação do cuidado; informação compartilhada, considerando a comunicação entre profissionais de saúde, pacientes e famílias de forma completa e imparcial, com informações recebidas em momento propício, efetivando sua participação no cuidado e na tomada de decisão; participação com os pacientes e famílias sendo incentivados e encorajados a participar do cuidado propi- ciando o seu empoderamento; e colaboração em que os pacientes e famílias são vistos como base de apoio à instituição, colaborando para o desenvolvimento, implantação e avaliação das políticas e programas que facilitam a prestação de cuidados à saúde e a educação profissional. ${ }^{(9-11)}$

À medida que as famílias no ambiente hospitalar advém de culturas cada vez mais diversas, os riscos de falhas na comunicação podem aumentar. Estudos ${ }^{(11-13)}$ demonstraram que a experiência dos pais na unidade neonatal, ainda, é permeada pela falta de poder, hierarquia entre pais e equipe de saúde e papel periférico dos pais. Aspectos que podem comprometer o estabelecimento de uma comunicação aberta com a família, com uso de linguagem compreensível, precisa e consistente. Ademais, dificulta o envolvimento da família no cuidado direto do seu filho. Estes fatores acarretam na intensificação do sofrimento vivenciado pelos pais.

Torna-se relevante repensar o modelo atual de atenção ao recém-nascido e família na unidade neonatal, a partir de uma assistência integral, humanizada, em que os pais sejam reconhecidos como os primeiros responsáveis pelo cuidado de seus filhos e possam participar do processo de tomada de decisão sobre o tratamento e o cuidado junto com a equipe de saúde em um processo de parceria.

Os pesquisadores deste estudo são enfermeiras pediatras e nos seus contextos de prática perceberam a carência de instrumentos objetivos que pudessem traduzir um dos pressupostos do cuidado centrado na família - informação compartilhada - em indicadores empíricos para serem medidos, analisados e utilizados como elementos direcionadores das mudanças no cuidado da família na unidade neonatal.

Em uma revisão integrativa ${ }^{(14)}$ sobre o constructo acesso à informação compartilhada com a família do recém-nascido na unidade neonatal foram identificados três temas representativos das necessidades da família: 1-Ser ouvida e valorizada em seu conhecimento: trata-se da necessidade da família de receber informação e estabelecer com a equipe um diálogo em que os seus sentimentos, necessidades e condições para que possa cuidar do filho na unidade neonatal sejam contemplados; 2 . Ser orientada sobre o cuidado do recém-nascido e o ambiente neonatal: que engloba a necessidade dos pais em serem informados sobre o ambiente de cuidado, os elementos assistenciais e as 
particularidades dos recém-nascidos; e 3. Ser informada em linguagem acessível sobre as condições do recém-nascido: que contém subitens sobre a necessidade da família em receber informações sobre as condições clínicas do recém-nascido de maneira que atenda às suas demandas, em quantidade e qualidade, atualizadas constantemente, com respeito ao seu tempo e sem sobrecarga de informações.

A partir desses resultados foi construído um instrumento para avaliar o acesso à informação pela família denominado de Instrumento de Medida da Percepção da Família sobre o Acesso à Informação na Unidade Neonatal (AICEF-UN). O instrumento elaborado consta de 25 afirmativas, dividido em três domínios. O escore das respostas de cada afirmativa (subitem) foi classificado em uma escala tipo Likert, com a graduação de nunca (0), muito raramente (1), raramente (2), quase sempre (3) e sempre (4). A totalização dos pontos é feita em cada subescala e ao final é somado o total de cada uma delas, dividindo pelo número de subitens. Para a análise do resultado da percepção da família sobre o acesso às informações compartilhadas considerando a escala como um todo, foram adotados os aspectos psicométricos: nunca tenho as informações compartilhadas (0); muito raramente tenho as informações compartilhadas (1); Raramente tenho as informações compartilhadas (2); Quase sempre tenho as informações compartilhadas (3); Sempre tenho as informações compartilhadas (4).

Para ser utilizado este instrumento precisava ser submetido a validação e análise psicométrica. O objetivo deste estudo foi realizar a validação do instrumento de percepção do acesso à informação compartilhada com a família do recém-nascido na unidade neonatal.

\section{Métodos}

Trata-se de uma pesquisa metodológica fundamentada nas etapas preconizadas por Pasquali: ${ }^{(15)}$ teórica, empírica e analítica.

A primeira versão do instrumento foi submetida à avaliação por um Comitê de Juízes especialistas no tema, composto por sete profissionais de saúde que atuavam no ensino ou em unidade de neonatologia por mais de três anos e que atenderam aos critérios de classificação de especialista segundo o Modelo de Validação de Fehring ${ }^{(16)}$ adaptado para este estudo, com pontuação igual ou superior a cinco.

O Comitê de Juízes avaliou o instrumento quanto a semântica, compreensão dos itens, redação e pertinência conceitual, utilizando a técnica Delphi. Um questionário foi preenchido pelos membros, com espaços para resposta de cada item em relação a sua relevância e para a emissão de sugestões.

A validação semântica do instrumento foi realizada com quinze (15) representantes de famílias da unidade neonatal, de um hospital de ensino da zona sul do município de São Paulo. Esta unidade dispõe de oito leitos de terapia intensiva e quatorze (14) leitos para cuidados semi-intensivos. A equipe de saúde é multiprofissional, composta por médicos neonatologistas, docentes de enfermagem e medicina, enfermeiras, técnicas de enfermagem, fisioterapeutas, psicólogo, nutricionista e alunos de pós-graduação de medicina, enfermagem, fisioterapia e fonoaudiologia.

Na unidade, já a alguns anos tem sido implementado o modelo do cuidado centrado no paciente com a sensibilização dos profissionais e adoção de boas práticas com famílias como o incentivo para sua participação junto com a equipe nas atividades diárias ligadas à higiene, curativo do coto umbilical, limpeza oral e ocular. Aliado à adoção da Estratégia do Método Canguru, formação de grupos de pais, e o atendimento individual às famílias em situações de crise, como o nascimento de crianças com malformação, agravamento das condições clínicas do RN, e em casos de indicação de cuidados paliativos de final de vida.

$\mathrm{Na}$ fase inicial da fase empírica, o instrumento foi aplicado a representantes de família com a realização do teste e re-teste, em uma amostra de vinte (20) participantes. A concordância das respostas foi obtida por meio do coeficiente de Kappa e a concordância entre os dois momentos, avaliada pelo coeficiente de correlação intraclasse (ICC). O coeficiente de Kappa foi de 0,87 demonstrando completa concordância e o ICC foi de 0,90 , o que demonstrou uma ótima estabilidade entre as duas avaliações.

A fase empírica ou experimental, foi uma etapa técnica de aplicação do instrumento piloto e da coleta das informações para que se procedesse à avaliação da qualidade psicométrica do instrumento. ${ }^{(15)}$ 
A fase analítica da pesquisa configurou-se pela análise das propriedades psicométricas e das análises estatísticas.

O cálculo amostral partiu da aplicação do instrumento em 35 participantes, sendo 15 no pré-teste e 20 no teste-reteste. Considerou-se a média de respostas no item 1 "Ser ouvida e valorizada em seu conhecimento", com um nível de confiança de $90 \%$ e um erro de 0,10, em que foi o mínimo de 46 participantes.

A amostra, da fase empírica, foi composta por cinquenta (50) representantes de famílias de recém-nascidos internados na unidade neonatal. $\mathrm{O}$ critério de inclusão adotado foi ser família de recém-nascidos internados na unidade há mais de 72 horas; e o de exclusão, ser representante da família com distúrbios emocionais e cognitivos autodeclarados que implicam em barreiras na comunicação.

Para a coleta de dados, na fase empírica foi utilizado um questionário contendo as variáveis sociodemográficas dos representantes das famílias, informações referentes aos recém-nascidos e o instrumento AICEF-UN.

As variáveis relacionadas à família foram: dados sociodemográficos do membro familiar quanto ao sexo, cor de pele, estado civil, nível de escolaridade, profissão, procedência, número de filhos, composição familiar, renda familiar (em salário mínimo) e religião adotada.

As variáveis relacionadas ao recém-nascido foram: realização de pré-natal, número de consultas, tipo de parto, idade gestacional ao nascimento, peso de nascimento, sexo do RN, nota de Apgar, diagnóstico médico, e tempo de internação do RN no momento da entrevista com os representantes de família.

$\mathrm{Na}$ associação das variáveis quantitativas com o escore geral do instrumento AICEF-UN, utilizou a Correlação de Pearson e o Teste de Correlação. Quando a correlação for positiva significa que à medida que uma variável aumenta seu valor, a outra correlacionada a esta, também aumenta proporcionalmente e, quando negativa, implica que as variáveis são inversamente proporcionais.

O Teste de Analysis of variance (ANOVA) foi utilizado como teste paramétrico e comparou a média do Escore Geral com as covariáveis qualitativas.

Para a comparação das respostas de cada item do instrumento AICEF-UN e o escore geral foi aplicado o teste não paramétrico de Wilcoxon.
O nível de significância adotado para os testes foi de $5 \%(\alpha=0,05)$ e o pacote estatístico utilizado foi o SPSS for Windows, versão 17.0 (SPSS Inc. Chicago, Illinois), Minitab 16 e Excel Office 2010.

$\mathrm{O}$ projeto atendeu aos princípios nacional e internacionalmente adotados com pesquisas com seres humanos; aprovado no Comitê de Ética e Pesquisa da instituição da qual os pesquisadores fazem parte, sob número 0416/11.

\section{Resultados}

O percentual de concordância obtido entre os juízes foi acima de $80 \%$, após duas etapas de consulta, gerando a versão III. A validação semântica do instrumento versão III foi realizada com quinze (15) representantes de famílias e demonstrou boa compreensão dos itens, clareza e afirmativas pertinentes ao momento vivido na unidade neonatal. Os aspectos apontados pelos participantes desta fase foram quanto às sugestões de modificações no texto e desmembramento do item "Fico satisfeita com a comunicação que estabeleço com a equipe quanto à qualidade e quantidade de informações recebidas", que foi acatado pelos pesquisadores. Assim, a versão IV do instrumento manteve os 25 itens. A análise da consistência interna do instrumento de AICEF-UN versão IV obteve um Alfa de Cronbach de 0,86 , denotando ótima consistência interna.

A realização da análise fatorial foi possível em vista do resultado do KMO de 0,649 e do teste de Bartlett, estes apresentaram-se significantes, ou seja, existe correlação entre os dados do instrumento analisado. $\mathrm{Na}$ análise fatorial, por meio do método de Componentes Principais e rotação Varimax, verificou-se que, as 25 questões do instrumento geraram oito (8) fatores, onde a variabilidade total explicada foi de $78,2 \%$, considerado um valor adequado, com valores próprios (Eigenvalues) maiores do que um (1). As cargas fatoriais para cada uma das questões do instrumento AICEF-UN, em cada um dos fatores demonstrou carga fatorial acima de 0,4 , o que permite a sua manutenção no instrumento analisado (Tabela 1). Quanto maior a carga fatorial da questão mais representativa para o fator ou domínio. No estudo, as cargas fatoriais menores foram no Fator 3 - questão $16(0,483)$; e Fator 5 - questão $2(0,499)$. 
Tabela 1. Carga fatorial das questões do Instrumento de Percepção do Acesso à Informação Compartilhada Equipe Família na Unidade Neonatal

\begin{tabular}{|c|c|c|c|c|c|c|c|c|}
\hline & Fator 1 & Fator 2 & Fator 3 & Fator 4 & Fator 5 & Fator 6 & Fator 7 & Fator 8 \\
\hline Q11 & 0,839 & & & & & & & \\
\hline Q10 & 0,835 & & & & & & & \\
\hline Q23 & 0,833 & & & & & & & \\
\hline Q14 & 0,782 & & & & & & & \\
\hline Q12 & 0,713 & & & & & & & \\
\hline Q13 & & 0,900 & & & & & & \\
\hline Q17 & & 0,733 & & & & & & \\
\hline Q1 & & 0,723 & & & & & & \\
\hline Q22 & & 0,690 & & & & & & \\
\hline Q9 & & 0,615 & & & & & & \\
\hline Q5 & & & 0,830 & & & & & \\
\hline Q15 & & & 0,762 & & & & & \\
\hline Q18 & & & 0,718 & & & & & \\
\hline Q16 & & & 0,483 & & & & & \\
\hline Q21 & & & & 0,752 & & & & \\
\hline Q4 & & & & 0,713 & & & & \\
\hline Q6 & & & & 0,703 & & & & \\
\hline Q19 & & & & & 0,850 & & & \\
\hline Q20 & & & & & 0,584 & & & \\
\hline Q2 & & & & & 0,499 & & & \\
\hline Q7 & & & & & & 0,844 & & \\
\hline Q3 & & & & & & 0,714 & & \\
\hline Q24 & & & & & & & $-0,789$ & \\
\hline Q8 & & & & & & & 0,649 & \\
\hline Q25 & & & & & & & & 0,811 \\
\hline
\end{tabular}

No fator 7 houve uma questão com sinal negativo, questão 24 com - 0,789 , que indica uma relação inversamente proporcional. A maioria dos representantes de famílias era procedente de São Paulo (72\%), do sexo feminino (86\%), com companheiro (62\%), de etnia branca (46\%), com ensino médio completo (56\%), com vínculo empregatício (54\%), tendo uma crença religiosa (86\%), a média de idade foi de 28,0 anos $( \pm 6,04)$, com número de filhos de $1,8( \pm 0,80)$, renda familiar de $2,4( \pm 1,2)$ salários mínimos e com a composição familiar média de 4,2 $( \pm 1,2)$ membros por núcleo familiar. Quanto às variáveis dos recém-nascidos (Tabela 2), as mães realizaram uma média de $7,6( \pm 3,1)$ consultas no pré-natal e a maioria das crianças nasceram de parto cesáreo (72\%), a média de peso foi de 2198 gramas $( \pm 896 \mathrm{~g})$, a maioria era do sexo masculino (58\%), a média da idade gestacional era de 34 semanas $( \pm 4,8)$, com média de Apgar no primeiro e quinto minuto de $7,6( \pm 1,4)$ e $8,8( \pm 0,8)$, respectivamente e tempo de internação em média de 37,2 dias $( \pm 35,2)$.

Com relação aos diagnósticos dos recém-nascidos, $62 \%$ apresentaram extremo/baixo peso, $48 \%$ pre-
Tabela 2. Perfil dos recém-nascidos internados na unidade neonatal

\begin{tabular}{lc}
\hline Variáveis neonatais & $\mathrm{f}(\%)$ \\
\hline Sexo & \\
$\quad$ Masculino & $29(58)$ \\
Feminino & $21(42)$ \\
Nascido de parto & \\
$\quad$ Cesáreo & $36(72)$ \\
$\quad$ Normal & $11(22)$ \\
Fórceps & $3(6)$ \\
Número de consultas realizadas pela mãe no pré-natal & $7,6( \pm 3,1)$ \\
Idade (dias) & $37,2( \pm 35,2)$ \\
Peso (gramas) & $2.198( \pm 896)$ \\
Apgar $1^{\circ}$ & $7,6( \pm 1,4)$ \\
Apgar $5^{\circ}$ & $8.8( \pm 0,8)$ \\
Idade gestacional (semanas) & $34( \pm 4,8)$ \\
Tempo de internação (dias) & $37,2( \pm 35,2)$ \\
\hline
\end{tabular}

maturidade, $20 \%$ anomalia cardíaca e $20 \%$ anomalia do Sistema Nervoso Central (SNC), 16\% apresentaram Síndrome do Desconforto Respiratório (SDR), 8\% possuíam outras anomalias genéticas, $6 \%$ eram pequenos para idade gestacional (PIG) e 6\% tinham hipoglicemia, $4 \%$ demonstraram anomalia gastrointestinal e $2 \%$ individualmente apresentaram icterícia $\mathrm{ABO}$, hipotermia e infecção neonatal. A medida da percepção do acesso à informação pela família na unidade neonatal investigada demonstrou que a família "quase sempre têm acesso à informação" sobre o cuidado com o recém-nascido, com escore médio de 3,37 (Tabela 3). Os itens com melhores escores ("quase sempre") foram demonstrados em 22 itens: "14- Recebo informações que me ajudam a compreender a situação de saúde do meu filho" (escore médio 3,78), “2- Eu consigo ser ouvida pela equipe de saúde em minhas dúvidas e questionamentos" (escore médio 3,76), "3- Tenho minhas necessidades atendidas quando recebo informações" (escore médio 3,72), "23- Eu recebo informações detalhadas sobre a condição clínica do meu filho" (escore médio 3,72), "5- Meu estresse e sofrimento reduzem quando tenho uma boa comunicação com a equipe de saúde" (escore 3,70), "11-Eu sou informada sobre os cuidados recebidos pelo meu filho nos diferentes momentos da internação (admissão, período de internação e alta)" (escore 3,70), "13- As informações recebidas ajudam-me a cuidar do meu filho" (escore 3,68), "7- Participo das tomadas de decisões sobre o tratamento e o cuidado do meu filho na unidade neonatal" (escore 3,66), "12- Tenho minhas dúvidas esclarecidas pela equipe de saúde" (escore 3,66), “20- As informa- 
ções sobre o meu filho são dadas sem pressa" (escore 3,62), "18-Eu recebo informações de forma simples e com linguagem de fácil entendimento" (escore 3,58), "15- Recebo informações adequadas ao meu grau de preocupação e às minhas necessidades" (escore 3,54), "1-Encontro na equipe de saúde alguém para conversar e expor minhas necessidades e sentimentos",. No item "10- Eu recebo informações em relação aos equipamentos, ao ambiente e a rotina da unidade pela equipe de saúde" e "17-Recebo orientações suficientes sobre os cuidados do meu filho durante a internação para que me sinta segura para levá-lo para casa" ambos obteve escore 3,52. Ainda, com escore quase sempre foi verificado nas questões: "4-Minha ansiedade aumenta quando a equipe informa-me sobre meu filho" (escore 3,50), "22- Percebo que as informações dos membros da equipe são semelhantes e complementares evitando contradições" (escore 3,46), " 8- O meu conhecimento é levado em consideração pela equipe de saúde" (escore 3,42), "6- Ao receber informações sinto que tenho o controle da situação vivenciada" (escore 3,38), "21- As informações recebidas ocorrem conforme o meu tempo impedindo que me sinta sobrecarregada" (escore 3,36), "16- Consigo participar das tomadas de decisões porque me sinto bem informada" (escore 3,32), e "19- Fico insatisfeita com a qualidade e quantidade de informações que recebo" (escore 3,22).

Apenas nos itens: "9- Os outros membros da minha família são envolvidos nas informações que recebemos da equipe de saúde" (escore 2,52) e item "25- Eu utilizo livros ou internet para conseguir informações sobre a doença" (escore 2,40) as famílias obtiveram escore mais baixo (raramente). $\mathrm{O}$ item com menor escore (muito raramente) foi o foi o "24- Eu recebo informações escritas" (escore médio 1,16).

$\mathrm{Na}$ análise descritiva das variáveis quantitativas, observou-se que a amostra possui uma baixa variabilidade, com coeficiente de variação $(\mathrm{CV})$ menor que $50 \%$, demonstrando que os dados são homogêneos. Na busca de correlação das variáveis com o escore geral do Instrumento de Percepção da Família sobre o Acesso à Informação Compartilhada Equipe - Família na Unidade Neonatal observou que somente ocorreu correlação com o número de filhos, onde o valor de 30,6\% demonstra que quanto maior a quantidade de filhos, maior é o

Tabela 3. Escore médio da percepção da família do recém-nascido sobre o acesso à informação compartilhada equipe - família na unidade neonatal por itens do instrumento

\begin{tabular}{|c|c|c|}
\hline \multicolumn{2}{|c|}{ Questões } & \multirow{2}{*}{$\begin{array}{c}\text { Escore médio } \\
3,52\end{array}$} \\
\hline 1 & Encontro na equipe de saúde alguém para conversar e expor minhas necessidades e sentimentos & \\
\hline 2 & Eu consigo ser ouvida pela equipe de saúde em minhas dúvidas e questionamentos & 3,76 \\
\hline 3 & Tenho minhas necessidades atendidas quando recebo informações & 3,72 \\
\hline 4 & Minha ansiedade aumenta quando a equipe me informa sobre meu filho & 3,50 \\
\hline 5 & Meu estresse e sofrimento reduzem quando tenho uma boa comunicação com a equipe de saúde & 3,70 \\
\hline 6 & Ao receber informações sinto que tenho o controle da situação vivenciada & 3,38 \\
\hline 7 & Participo das tomadas de decisões sobre o tratamento e o cuidado do meu filho na unidade neonatal & 3,66 \\
\hline 8 & 0 meu conhecimento é levado em consideração pela equipe de saúde & 3,42 \\
\hline 9 & Os outros membros da minha família são envolvidos nas informações que recebemos da equipe de saúde & 2,52 \\
\hline 10 & Eu recebo informações em relação aos equipamentos, ao ambiente e a rotina da unidade pela equipe de saúde. & 3,52 \\
\hline 11 & Eu sou informada sobre os cuidados recebidos pelo meu filho nos diferentes momentos da internação (admissão, período de internação e alta) & 3,70 \\
\hline 12 & Tenho minhas dúvidas esclarecidas pela equipe de saúde & 3,66 \\
\hline 13 & As informações recebidas ajudam-me a cuidar do meu filho & 3,68 \\
\hline 14 & Recebo informações que me ajudam a compreender a situação de saúde do meu filho & 3,78 \\
\hline 15 & Recebo informações adequadas ao meu grau de preocupação e às minhas necessidades & 3,54 \\
\hline 16 & Consigo participar das tomadas de decisões porque me sinto bem informada & 3,32 \\
\hline 17 & Recebo orientações suficientes sobre os cuidados do meu filho durante a internação para que me sinta segura para levá-lo para casa & 3,52 \\
\hline 18 & Eu recebo informações de forma simples e com linguagem de fácil entendimento & 3,58 \\
\hline 19 & Fico insatisfeita com a qualidade e quantidade de informações que recebo & 3,22 \\
\hline 20 & As informações sobre o meu filho são dadas sem pressa & 3,62 \\
\hline 21 & As informações recebidas ocorrem conforme o meu tempo impedindo que me sinta sobrecarregada & 3,36 \\
\hline 22 & Percebo que as informações dos membros da equipe são semelhantes e complementares evitando contradições & 3,46 \\
\hline & Eu recebo informações detalhadas sobre a condição clínica do meu filho & 3,72 \\
\hline & Eu recebo informações por escrito & 1,16 \\
\hline & Eu utilizo livros ou internet para conseguir informações sobre a doença & 2,40 \\
\hline Esc & re geral & 3,37 \\
\hline
\end{tabular}


Escore Geral, isto é melhor o acesso à informação. No entanto, essa correlação é considerada ruim. Ao correlacionar as variáveis qualitativas com o Escore Geral do AICEF-UN (Tabela 4) verificou-se que não houve diferença estatística, com exceção da cor de pele negra, que demonstrou um valor de $\mathrm{p}$ significante $(0,033)$.

Tabela 4. Correlação com o Escore Geral do Instrumento de Medida da Percepção da Família sobre o Acesso à Informação na Unidade Neonatal

\begin{tabular}{|c|c|c|}
\hline Escore Geral & Corr & $p$-value \\
\hline Idade & $-15,3 \%$ & 0,289 \\
\hline$N^{0}$ filhos & $30,6 \%$ & 0,031 \\
\hline $\mathrm{N}^{0}$ familiares & $12,4 \%$ & 0,391 \\
\hline Renda & $0,3 \%$ & 0,981 \\
\hline $\mathrm{N}^{0}$ Consultas & $20,7 \%$ & 0,149 \\
\hline Peso & $-4,3 \%$ & 0,767 \\
\hline Apgar $1^{\circ}$ & $-8,3 \%$ & 0,565 \\
\hline Apgar $5^{\circ}$ & $-5,3 \%$ & 0,716 \\
\hline Idade Gestacional & $3,0 \%$ & 0,837 \\
\hline Tempo de internação (dias) & $-10,2 \%$ & 0,480 \\
\hline
\end{tabular}

\section{Discussão}

A permanência $24 \mathrm{~h}$ dos pais na unidade neonatal hoje demanda uma nova maneira de organização da assistência por parte da equipe de saúde. É preciso olhar de maneira mais humanizada e não automatizada para o cuidado, estreitando as relações entre equipe de saúde e família envolvendo-a no processo de cuidar, como parceira.

A validação do Instrumento de Percepção da Família sobre o Acesso à Informação Compartilhada Equipe - Família na Unidade Neonatal- AICEF-UN seguiu todas as etapas de validação de escalas psicométricas proposta por Pasquali, ${ }^{(14,15)}$ e pelas organizações American Educational Research Association, American Psychological Association and the National Council on Measurement in Education, representando o padrão ouro na orientação sobre testes nos Estados Unidos e muitos outros países. ${ }^{(17)}$

Os resultados apresentados evidenciaram que o instrumento obteve ótima consistência interna, ou seja, os itens poderão ser utilizados em qualquer ocasião de uso do teste. ${ }^{(14)}$

Na validação clínica do instrumento AICEF-UN, foi identificado que a família percebe "quase sempre" recebe informações que a ajudam na compreensão da situação vivida. Pode-se inferir, esse achado, à um movimento dos profissionais da referida unidade neonatal em atender ao preconizado pelo modelo do CCPF, considerando a implementação vem sendo realizada.

Na literatura ${ }^{(18)}$ destaca-se o compartilhamento de informações como elemento central para que a família seja capaz de enfrentar a situação de forma menos traumática. Os autores apontam a falta de informações como fator que agrava o sentimento de desespero da família e evidenciam que após a implantação de um protocolo de acolhimento aos pais, os mesmos se tornaram mais empoderados para agir.

No entanto, é preciso levar em consideração que nem sempre os profissionais oferecem informações, indo em direção contrária ao preconizado pelo MCCPF. Como destacado no estudo ${ }^{(19)}$ em que os autores apontam a falta de esclarecimento à família no momento da internação. Eles reconhecem que é preciso ter sensibilidade para fornecer as informações, pois a família não dispõe do mesmo saber que o profissional, mas não validam o entendimento dos pais.

O sucesso de uma comunicação efetiva está baseado no diálogo, seguindo as etapas de perguntar, ouvir, explicar, advogar, verbalizar e negociar, objetivando alcançar a compreensão mútua do assunto. ${ }^{(20)}$

Nesta pesquisa, a família percebe que consegue ser ouvida, expor suas dúvidas e questionamentos, o que consequentemente pode contribuir para a redução do estresse vivido. A família precisa ser encorajada e incentivada a participar do cuidado ao recém-nascido, mas para isso a equipe de profissionais da unidade neonatal deve proporcionar informações com uma linguagem simples, clara e objetiva em relação ao ambiente, equipamentos e ao estado de saúde do recém-nascido, além de auxiliarem nas dificuldades encontradas pelos pais no momento do cuidado e durante toda a internação.

No entanto, as famílias indicaram que quase sempre não apresentam dúvidas em relação aos equipamentos, ao ambiente e as rotinas da unidade neonatal, e que as informações que são recebidas auxiliam na participação do cuidar. Também apontaram que quase sempre participam nas tomadas de decisão sobre o tratamento do RN. Isto nos indica que é preciso melhorar ainda mais a maneira como as informações chegam até as famílias, buscando maior aproximação com a realidade que cada uma apresenta, de maneira singular. 
Em contraste com os resultados dessa pesquisa, estudos $^{(7,21,22)}$ revelam que os profissionais ainda não consideram a família como parceira do cuidado. Os autores $^{(21)}$ mostram que a participação dos pais nos cuidados dos filhos ainda é segregada na prática, sendo condicionada ao grau de gravidade do RN. Já no estudo, ${ }^{(7)}$ os autores revelam que a participação dos pais no cuidado do bebê na unidade neonatal é permitida para as atividades de higiene e alimentação do neonato. No entanto, a participação ou presença dos pais durante procedimentos é mínima, sendo permitida apenas em alguns casos ou, até mesmo, não sendo permitida em nenhuma ocasião.

Outro aspecto evidenciado neste estudo é que, outros membros da família não foram envolvidos nas informações fornecidas pela equipe de saúde, limitando esse acesso somente aos pais ou ao cuidador responsável.

Em um estudo, os autores enfatizam que a separação entre o RN e a família ocasionada pela internação, pode prejudicar a formação do vínculo afetivo. Nesse período todos os membros da família vivenciam o sofrimento e as preocupações pela saúde do RN. Por esse motivo, a assistência da equipe de saúde deve ser estendida a todos os membros da família.

No contexto da unidade neonatal, a situação dos pais, avós e irmãos é mais desfavorável do que a da mãe do recém-nascido sendo inquestionável o papel de destaque que a mãe ocupa na composição familiar, no entanto, não se pode ignorar o papel e necessidades dos outros componentes da família. ${ }^{(7)}$

Neste estudo, as famílias percebem que muito raramente recebem informações detalhadas e escritas sobre seus filhos, e raramente buscam informações em livros ou na internet, corroborando achados de outros estudos $^{(6,20)}$ em que, apesar das famílias receberem informações detalhadas, raramente elas a recebem por escrito; a maior preferência é obtê-las diretamente com a equipe de saúde, de forma mais objetiva quanto ao real estado de saúde dos seus filhos.

Em estudo brasileiro sobre informações em unidades neonatais, o resultado evidencia que as informações acessadas pelas famílias são muitas vezes superficiais e ineficazes para a sua segurança na continuidade do cuidado do filho no domicílio. Os pais não percebem as conversas do dia a dia como orientações, nem o preparo para alta hospitalar, e ainda mais preocupante é o fato de receberem informações de outros pais. As orientações são realizadas sempre de forma verbal, mas a equipe de enfermagem reconhece que a existência de um material com informações e orientações por escrito facilitaria a compreensão dos pais.(23)

O CCPF otimiza as relações entre profissionais de saúde e familiares e favorece a construção do vínculo com o RN. Com a redução do estresse da família, a experiência de hospitalização é melhorada e o desenvolvimento de uma comunicação eficaz reduz os conflitos da relação, incluindo ações judiciais. ${ }^{(24,25)}$

A prática do CCPF poderá ser implantada quando a equipe de saúde reconhecer o papel que as famílias desempenham como principal responsável pela saúde e o bem-estar das crianças e membros familiares. É fundamental que se reconheça que o apoio emocional, social e de desenvolvimento são componentes integrais nos cuidados em saúde.

No entanto, mesmo conhecendo todos os benefícios desse modelo de cuidado, sua implantação na prática, ainda é um desafio, principalmente quanto ao pressuposto de colaboração. Ainda assim, fica evidente que a comunicação favorece as relações interpessoais e constitui o ponto chave para o sucesso da implantação do CCPF. ${ }^{(25)}$

O estudo realizado aponta que ainda muito se requer de investimento para que o acesso à informação pela família seja garantida nas instituições hospitalares, requerendo mudança da cultura organizacional e formação da equipe multiprofissional para o atendimento dos pressupostos do CCPF que são dignidade e respeito, informação compartilhada, colaboração, participação.

\section{Conclusão}

O Instrumento de Percepção da Família sobre o Acesso à Informação compartilhada Equipe - Família na Unidade Neonatal apresentou ótima consistência. Os dados apresentaram um resultado de KMO e teste de Bartlett significantes, demonstrando correlação dos itens com o constructo analisado pelo instrumento. $\mathrm{Na}$ análise Fatorial, os instrumentos geraram oito fatores e com cargas fatoriais que permitiram a manutenção no instrumento. Houve correlação significante da variável número de filhos com o escore geral do instrumento, quanto maior a quantidade de filhos maior foi o acesso à informação; e do escore geral do com a 
cor de pele negra. A família percebeu que quase sempre têm acesso à informação na unidade neonatal e muito raramente recebe informações detalhadas e escritas sobre o filho; o que requer investimento na mudança da cultura organizacional para a valorização e inclusão da família como centro do cuidado de enfermagem e melhoria no processo de comunicação.

\section{Contribuições}

Boyamian TMDL, Mandetta MA, Silva SQ, Damaceno DF, Oliveira TS, Balbino FS e Balieiro MMFG declaram que contribuíram com a concepção do estudo, análise e interpretação dos dados, redação do artigo, revisão crítica relevante do conteúdo intelectual e aprovação da versão final a ser publicada.

\section{Referências}

1. Atashi V, Kohan S, Salehi Z, Salehi K. Maternal-fetal emotional relationship during pregnancy, its related factors and outcomes in Iranian pregnant women: a panel study protocol. Reprod Health. 2018;15:176.

2. Exequiel NP, Milbrath VM, Gabatz RI, Vaz JC, Silva LL, Klumb MM, et al. Sentimentos vivenciados pelas mães na hospitalização neonatal. Enferm Foco. 2021;12(1):73-8.

3. Ahlqvist-Björkroth $\mathrm{S}$. Communication between healthcare professionals and parents is a keyfactor in involving parents in neonatal intensive care [editorial]. Acta Paediatr. 2018;107(1):12-3.

4. Hall SL, Hynan MT, Phillips R, Lassen S, Craiag JW, Goyer E, et al. The neonatal intensive parenting unit: an introduction. J Perinatol. 2017;37:1259-64.

5. Pineda R, Bender J, Hall B, Shabosky L, Annecca A, Smith J. Parent participation in the neonatal intensive care unit: Predictors and relationships to neurobehavior and developmental outcomes. Early Hum Dev. 2018;117:32-8.

6. Altimier L, Phillips R. The Neonatal Integrative Developmental Care Model: Advanced Clinical Applications of the Seven Core Measures for Neuroprotective Family-centered Developmental Care. Newborn Infant Nurs Ver. 2016;16:230-44.

7. Bueno MT, Quiroga A, Rodríguez S, Sola A. Acceso de las familias a las unidades de internación de Neonatología en Iberoamérica: una realidad a mejorar. An Pediatr. 2015;85(2):95-101.
8. Park M, Giap $\Pi$, Lee $M$, Jeong $H$, Jeong $M$, Go $Y$. Patient- and family-centered care interventions for improving the quality of health care: A review of systematic reviews. International. Int J Nurs Stud. 2018, 87:69-83.

9. Gasparini R, Champagne M, Stephany A, Hudson J, Fuchs MA. Policy to practice: increased family presence and the impact on patient and family centered care adoption. J Nurs Adm. 2015;45(1):28-34.

10. Partnering with patients and families to design a patient-and family-centered health care system: recommendations and promising practices. 2a ed. Cambridge: Institute for FamilyCentered Care; 2011.

11. Banerjee J, Aloysis A, Platonos AD. Family centred care and family delivered care - What are we talking about? J. Neonatal Nurs. 2018;24(1):8-12.

12. Serlachius A, Hames J, Juth V, Garton D, Rowley S, Petrie KJ. Parental experiences of familycentred care from admission to discharge in the neonatal intensive care unit. J Paediatr Child Health. 2018;54(11):1227-33.

13. Benzies KM, Shah V, Aziz K, Lodha A, Misfeldt R. The health care system is making 'too much noise' to provide family-centred care in neonatal intensive care units: Perspectives of health care providers and hospital administrators. Intensive Crit Care Nurs. 2019;50:44-53.

14. Damaceno DF. Construção de indicadores empíricos sobre a informação compartilhada entre a equipe de saúde e família na unidade neonatal [trabalho de conclusão de curso]. São Paulo: Universidade Federal de São Paulo; 2011.

15. Pasquali L. Psicometria. Rev Esc Enferm USP. 2009;43(Esp):992-9.

16. Fehring RJ. The Fehring model. In: Carrol-Jonhnson RM, Paquete M, editores. Classification of nursing diagnoses: proceedings of the Tenth Conference; 1994. Philadelphia: J.B. Limppincott; 1994. p. 55-62.

17. American Psychological Association (APA). The Standards for Educational and Psychological Testing. Washingto: Aera Publications; 2014.

18. Soares LG, Soares LG, Decesaro MN, Higarasho IH. Percepção das famílias sobre 0 acolhimento no contexto neonatal durante um processo de intervenção. Rev Fun Care Online. 2019;11(1):147-53.

19. Caneco E0, Milbrath VM, Freitag VL, Amestoy SC. Cuidado prestado pela equipe de saúde às famílias de crianças que sofreram Asfixia Perinatal Grave. Rev Saúde (St. Maria). 2017;43(3):1-10.

20. Giambra BK, Broome ME, Sabourin T, Buelow J, Stiffler D. Integration of Parent and Nurse Perspectives of Communication to Plan Care for Technology Dependent Children: The Theory of Shared Communication. J Pediatr Nurs. 2017;34:29-35.

21. Rodrigues BC, Uema RT, Rissi GP, Felipin LC, Higarashi IH. Cuidado centrado na família e sua prática na unidade de terapia intensiva neonatal. Rev Rene. 2019;20:e39767.

22. Lima VF, Mazza VA, Mor LM, Pinto MN. Vivência dos familiares de prematuros internados em unidade de terapia intensiva neonatal. Rev Min Enferm. 2017;21:e-1026.

23. Saldanha MD, Borio TC, Gabatz RI, Milbrath VM, Vaz JC. Information about the hospital in the neonatal intensive therapy unit: perspective of the parents. Rev Enferm UFPI. 2018;7(4):22-8.

24. Wells N, Bronheim S, Zyzanski S, Hoover C. Psychometric Evaluation of a Consumer-Developed Family-Centered Care Assessment Tool. Matern Child Health J. 2005;19(9):1899-909.

25. Hill C, Knafl KA, Santacroce SJ. Family-Centred Care From the perspective of parents of children cared for in a Pediatric Intensive Care Unit: an integrative review. J Pediatr Nurs. 2018;41:22-33. 